IUHET 513, September 2007

\title{
PERSPECTIVES ON LORENTZ AND CPT VIOLATION
}

\author{
V. ALAN KOSTELECKÝ \\ Physics Department, Indiana University \\ Bloomington, IN 47405, U.S.A.
}

This talk offers some comments and perspectives on Lorentz and CPT violation.

\section{Introduction}

Lorentz symmetry is the invariance of physical laws under rotations and boosts. As a global symmetry over Minkowski spacetime, it underlies the theory of Special Relativity and the Standard Model of particle physics, where it is intimately tied to CPT invariance. As a local symmetry of freely falling frames, it is an essential component of General Relativity. Nonetheless, the possibility exists that nature may exhibit tiny violations of Lorentz symmetry. This talk presents some perspectives on the basic motivations and reasoning in this subject.

Since Lorentz symmetry has been verified in many experiments, as has $\mathrm{CPT}$ invariance, it is reasonable to ask why relativity violations are worth considering. A sufficient theoretical motivation is the need for a consistent description of Lorentz and CPT violation to offer guidance for experimental tests. However, a stronger motivation is the prospect that Lorentz and CPT violation can serve as a sensitive potential signal for physics at the Planck scale. In fact, the present interest in the subject was triggered by the realization that natural mechanisms for Lorentz and CPT violation exist in unified theories at the Planck scale. ${ }^{1}$ The large range of existing phenomenological and experimental activities stems from the application of effective field theory ${ }^{2}$ and the construction of the Standard-Model Extension $(\mathrm{SME})^{3,4}$ to catalogue and predict observable effects.

\section{Approaches and origins}

The study of Lorentz and CPT violation can be approached on three distinct levels. First, at the level of fundamental theory at the unification 
scale, one can investigate possible mechanisms and determine their features and implications. Second, at the level of theory at accessible scales, one can seek a description of the resulting effects that is quantitative, general, and compatible with the established physics of the Standard Model and General Relativity. Finally, at the level of observation and experiment, one can study and perform both high sensitivity tests and broad searches, preferably ones that are feasible with existing or near-future technology.

The first point to establish is whether Lorentz violation can indeed occur in a fundamental theory. Without at least one viable mechanism, the interest of the idea would be much reduced. A plausible origin for Lorentz violation has been identified in string field theory, ${ }^{1}$ which has interactions with a generic structure that could in principle trigger spontaneous violation of Lorentz symmetry and generate vacuum expectation values for Lorentz tensor fields. More recently, numerous other mechanisms for Lorentz violation at the fundamental level have been proposed including, for example, ones involving noncommutative field theories, ${ }^{5}$ spacetime-varying fields, ${ }^{6}$ quantum gravity, ${ }^{7}$ random-dynamics models, ${ }^{8}$ multiverses,${ }^{9}$ brane-world scenarios,${ }^{10}$ supersymmetry, ${ }^{11}$ and massive gravity. ${ }^{12}$

Given that mechanisms exist for Lorentz and CPT violation in an underlying theory, it is natural to ask about the consequences for physics at observable scales. In particular, the question arises as to the best method to develop a description of the possible effects.

\section{Describing Lorentz violation}

Some key criteria offer valuable guidance in the search for a suitable theoretical framework for describing Lorentz violation at attainable scales. The first is coordinate independence. It has been accepted since long before 1905 that the physics of a system should be independent of a change of observer coordinates. This holds whether a coordinate change is implemented via a Lorentz transformation or in any other way.

The second is realism. Since 1905, when virtually no fundamental particles were known and quantum physics was at its dawn, thousands of people have invested millions of person-hours and billions of dollars in establishing the Standard Model of particle physics and General Relativity as an accurate description of nature. To be of real interest nowadays, any proposed theoretical framework for Lorentz violation must incorporate this well-established physics.

The third is generality. No compelling evidence for Lorentz violation 
exists at present. Physics is therefore currently in the position of searching for a violation, as opposed to attempting to understand an observed effect. In the searching phase, it is desirable to have the most general possible formulation so that no region is left unexplored. This is in strong contrast to the modeling phase, where considerations such as simplicity are important in attempts to understand a known effect.

Armed with these criteria, we can follow the basic reasoning that leads to the application of effective field theory and the construction of the SME.

\subsection{Modified Lorentz transformations}

Since the essential content of Special Relativity is the idea that physics is invariant under Lorentz transformations, the most obvious approach to describing relativity violations is to investigate modifications of the Lorentz transformations. In fact, the literature since 1905 abounds with various ad hoc proposals of this type. However, independent of any specific proposal, this approach has some serious disadvantages.

One is that a textbook Lorentz transformation acts on the observer and therefore corresponds merely to a change of coordinates, i.e., a change of reference frame. However, according to the above criterion of coordinate independence, a frame change cannot have physical implications by itself. The key feature of Special Relativity is really the requirement that the equations for the system being observed must be covariant under a Lorentz transformation, which intrinsically assumes that Lorentz symmetry is exact. This approach is therefore problematic for investigating violations.

It is of course possible to construct special models imposing form covariance of the system under some ad hoc alternative transformation. However, any specific such proposal runs counter to the criterion of generality. Moreover, some kinds of violations are difficult and perhaps even impossible to countenance via this approach. For example, Lorentz violation in nature might well be particle-species dependent, but it is very challenging to formulate a description of this flavor dependence based on modified Lorentz transformations of the observer. The criterion of realism presents a further substantial obstacle, since it is awkward at best to implement such models in the context of the Standard Model and General Relativity.

\subsection{Modified dispersion laws}

The above discussion suggests that a general and realistic investigation of Lorentz violation is most naturally performed directly in terms of the 
properties of a system rather than via modifications of the Lorentz transformations. A simple implementation of this is to study modifications of particle dispersion laws. However, this also suffers from serious drawbacks.

One issue involves the criterion of generality. Modifications of dispersion laws can only describe changes in the free propagation of particles and perhaps also partially account for interaction kinematics. However, physics is far more than free propagation, and this approach therefore disregards a large range of interesting Lorentz-violating effects involving interactions.

There are also various issues associated with the choice of modifications to the dispersion law. Not all choices are compatible with desirable features such as originating from an action. Also, meaningful physical measurements must necessarily compare two quantities, so some choices may be unphysical. In particular, calculations with a modified dispersion law that yield apparent changes of properties are insufficient by themselves to demonstrate physical Lorentz violation. A simple example of a modified dispersion law with no observable consequences in Minkowski spacetime is ${ }^{3} p^{\mu} p_{\mu}=m^{2}+a_{\mu} p^{\mu}$, where $a_{\mu}$ is a prescribed set of four numbers in a given frame. Direct calculations with this dispersion law appear to give Lorentz-violating properties that depend on the preferred vector $a_{\mu}$, but in fact they are unobservable because $a_{\mu}$ can be eliminated via a physically irrelevant redefinition of the energy and momentum. The observability of modifications to a dispersion law can be challenging to demonstrate.

\subsection{Effective field theory and the $S M E$}

We see that the desiderata for a satisfactory description of Lorentz violation include a comprehensive treatment of free and interacting effects in all particle species. Remarkably, a model-independent and general approach of this type exists.

The key is to take advantage of the idea that Lorentz violation at attainable energies can be described using effective field theory, independent of the underlying mechanism. ${ }^{2,13}$ Starting from the Standard Model coupled to General Relativity, we can add to the action all possible scalar terms formed by contracting operators for Lorentz violation with coefficients that control the size of the effects. The operators are naturally ordered according to their mass dimension. The resulting realistic effective field theory is the SME. ${ }^{3,4}$ Since CPT violation in realistic field theories comes with Lorentz violation, ${ }^{14}$ the SME also incorporates general CPT violation.

By virtue of its construction, the SME satisfies the three guiding crite- 
ria of coordinate invariance, realism, and generality. Moreover, it handles simultaneously all particle species, including both propagation and interaction properties, so its equations of motion contain all action-compatible modifications of realistic dispersion laws. The coordinate invariance implies that physics is unaffected by observer frame changes, including Lorentz and other transformations, while particle transformations can produce observable effects of Lorentz violation.

The primary disadvantage of the SME approach is its comparative complexity and the investment required to become proficient with its use. However, this is outweighed by its advantages as a realistic, general, and calculable framework for describing Lorentz violation. Judicious choices establishing relations among the SME coefficients for Lorentz violation yield elegant and simple models that can serve as a theorist's playground, while the general case offers guidance for broad-based experimental searches.

\section{Gravity and Lorentz violation}

The SME allows for both global ${ }^{3}$ and local ${ }^{4}$ Lorentz violation, and interesting effects arise from local Lorentz violation in the gravitational context. In general, local Lorentz violation can be understood as arising when a nonzero coefficient $t_{a b c \ldots}$ for Lorentz violation exists in local freely falling frames. ${ }^{4}$ The cofficient $t_{a b c \ldots}$ can be converted to a coefficient $t_{\lambda \mu \nu \ldots}$ on the spacetime manifold using the vierbein $e_{\mu}{ }^{a}$.

One result is that spontaneous violation of local Lorentz symmetry is always accompanied by spontaneous diffeomorphism violation, and vice versa. ${ }^{15} \mathrm{~A}$ nonzero coefficient $t_{a b c \ldots}$ is the vacuum value of a local Lorentz tensor field, and it implies Lorentz violation because it is invariant instead of transforming like a tensor under particle transformations. The vierbein ensures that there is a corresponding spacetime tensor field with vacuum value $t_{\lambda \mu \nu \ldots}$ on the spacetime manifold, which in turn implies spontaneous diffeomorphism breaking because it is invariant instead of transforming like a tensor under particle diffeomorphisms.

A more surprising result is that explicit Lorentz violation is generically incompatible with Riemann geometry. ${ }^{4}$ Explicit violation occurs when the SME coefficients are externally prescribed, but the ensuing equations of motion turn out to be inconsistent with the Bianchi identities. This result also holds in Riemann-Cartan spacetime. However, spontaneous violation evades the difficulty because it generates the SME coefficients dynamically, thereby ensuring compatibility with the underlying spacetime geometry. 
Spontaneous local Lorentz violation is accompanied by up to $10 \mathrm{Nambu-}$ Goldstone (NG) modes. ${ }^{15}$ With a suitable choice of gauge, these modes can be identified with components of the vierbein normally associated with local Lorentz and diffeomorphism gauge freedoms. The physical role of the NG modes varies, but in general they represent long-range forces that can be problematic for phenomenology. However, in certain models the NG modes can be interpreted as photons, thus offering the intriguing prospect that the existence of light could be a consequence of Lorentz violation instead of local $\mathrm{U}(1)$ gauge invariance, with concomitant observable signals. ${ }^{15,16}$ A similar interpretation is possible for the graviton. ${ }^{17}$ Other potential experimental signals arise from NG modes in the gravity ${ }^{18}$ and matter ${ }^{19}$ sectors, and from torsion. ${ }^{20}$ The spectrum of vacuum excitations typically also includes massive modes that may lead to additional observable effects. ${ }^{21}$

\section{The search for signals}

The SME predicts some unique signals, such as rotational, sidereal, and annual variations. The effects are likely to be heavily suppressed, perhaps as some power of the ratio of an accessible scale to the underlying scale, but they could be detected using sensitive tools such as interferometry. For example, meson interferometry offers the potential to identify flavorand direction-dependent energy shifts of mesons relative to antimesons, ${ }^{22}$ while exquisite interferometric sensitivity to polarization-dependent effects of photons is attained using cosmological birefringence. ${ }^{23}$ Conceivably, SME effects might even be reflected in existing data, such as those for flavor oscillations of neutrinos. ${ }^{24}$ Overall, an impressive range of sensitivities in the matter, gauge, and gravitational sectors of the SME has been achieved. ${ }^{25}$

Despite a decade of intense activity, most of the SME coefficient space is still unexplored by experiments, and many basic theoretical issues are unaddressed. The study of relativity violations remains fascinating, with the enticing prospect of identifying a signal from the Planck scale.

\section{Acknowledgments}

This work was supported in part by DoE grant DE-FG02-91ER40661 and NASA grant NAG3-2914.

\section{References}

1. V.A. Kostelecký and S. Samuel, Phys. Rev. D 39, 683 (1989); V.A. Kostelecký and R. Potting, Nucl. Phys. B 359, 545 (1991). 
2. V.A. Kostelecký and R. Potting, Phys. Rev. D 51, 3923 (1995).

3. D. Colladay and V.A. Kostelecký, Phys. Rev. D 55, 6760 (1997); Phys. Rev. D 58, 116002 (1998).

4. V.A. Kostelecký, Phys. Rev. D 69, 105009 (2004).

5. See, for example, I. Mocioiu et al., Phys. Lett. B 489, 390 (2000); S.M. Carroll et al., Phys. Rev. Lett. 87, 141601 (2001).

6. V.A. Kostelecký et al., Phys. Rev. D 68, 123511 (2003).

7. See, for example, G. Amelino-Camelia et al., AIP Conf. Proc. 758, 30 (2005); N.E. Mavromatos, Lect. Notes Phys. 669, 245 (2005); Y. Bonder and D. Sudarsky, arXiv:0709.0551.

8. C.D. Froggatt and H.B. Nielsen, hep-ph/0211106.

9. J.D. Bjorken, Phys. Rev. D 67, 043508 (2003).

10. See, for example, C.P. Burgess et al., JHEP 0203, 043 (2002).

11. M. Berger and V.A. Kostelecký, Phys. Rev. D 65, 091701(R) (2002); P.A. Bolokhov et al., Phys. Rev. D 72, 015013 (2005).

12. See, for example, G. Dvali et al., Phys. Rev. D 76, 044028 (2007); D.S. Gorbunov and S.M. Sibiryakov, JHEP 0509, 082 (2005); M.V. Libanov and V.A. Rubakov, JHEP 0508, 001 (2005); N. Arkani-Hamed et al., JHEP 0507, 029 (2005); V.A. Kostelecký and S. Samuel, Phys. Rev. D 42, 1289 (1990); Phys. Rev. Lett. 66, 1811 (1991).

13. V.A. Kostelecký and R. Lehnert, Phys. Rev. D 63, 065008 (2001).

14. O.W. Greenberg, Phys. Rev. Lett. 89, 231602 (2002).

15. R. Bluhm and V.A. Kostelecký, Phys. Rev. D 71, 065008 (2005).

16. B. Altschul and V.A. Kostelecký, Phys. Lett. B 628, 106 (2005).

17. V.A. Kostelecký and R. Potting, Gen. Rel. Grav. 37, 1675 (2005).

18. Q.G. Bailey and V.A. Kostelecký, Phys. Rev. D 74, 045001 (2006).

19. V.A. Kostelecký and J.D. Tasson, in preparation.

20. V.A. Kostelecký, N. Russell, and J.D. Tasson, arXiv:0712.4393.

21. R. Bluhm et al., arxiv:0712.4119; V.A. Kostelecký and S. Samuel, Phys. Rev. D 40, 1886 (1989); Phys. Rev. Lett. 63, 224 (1989).

22. H. Nguyen (KTeV), hep-ex/0112046; A. Di Domenico et al. (KLOE), these proceedings; B. Aubert et al. (BaBar), hep-ex/0607103; arXiv:0711.2713; D.P. Stoker (BaBar), these proceedings; J.M. Link et al. (FOCUS), Phys. Lett. B 556, 7 (2003); V.A. Kostelecký, Phys. Rev. Lett. 80, 1818 (1998); Phys. Rev. D 61, 016002 (2000); Phys. Rev. D 64, 076001 (2001).

23. V.A. Kostelecký and M. Mewes, Phys. Rev. Lett. 87, 251304 (2001); Phys. Rev. D 66, 056005 (2002); Phys. Rev. Lett. 97, 140401 (2006); Phys. Rev. Lett. 99, 011601 (2007).

24. L.B. Auerbach et al., Phys. Rev. D 72, 076004 (2005); B.J. Rebel and S.F. Mufson, these proceedings; V.A. Kostelecký and M. Mewes, Phys. Rev. D 69, 016005 (2004); Phys. Rev. D 70, 031902(R) (2004); Phys. Rev. D 70, 076002 (2004); T. Katori et al., Phys. Rev. D 74, 105009 (2006); V. Barger et al., Phys. Lett. B 653, 267 (2007); K. Whisnant, these proceedings.

25. Results are tabulated in V.A. Kostelecký and N. Russell, arXiv:0801.0287. 